\title{
THE MERCEDES-BENZ APPROACH TO $\gamma$-RAY ASTRONOMY
}

\author{
Carl W. AKERLOF \\ Randall Laboratory of Physics, University of Michigan, Ann Arbor, Michigan 48109, USA
}

\begin{abstract}
The sensitivity requirements for ground-based $\gamma$-ray astronomy are reviewed in the light of the most reliable estimates of stellar fluxes above $100 \mathrm{GeV}$. Current data strongly favor the construction of detectors with the lowest energy thresholds. Since improvements in angular resolution are limited by shower fluctuations, better methods of rejecting hadronic showers must be found to reliably observe the known astrophysical sources. Several possible methods for reducing this hadronic background are discussed.
\end{abstract}

The name "Mercedes-Benz" is internationally famous for automotive products that deliver high performance at a non-negligible cost. Given the present ambiguous state of ground-based $\gamma$-ray astronomy, I think this might be a useful theme for the next generation of detectors. Without some significant innovations in technique and equipment, this field will not achieve the decisive role in probing high energy astrophysical processes that many have anticipated. Unfortunately such improvements will not come cheaply and the resources of our funding agencies for this activity are significantly limited.

The design of a new $\gamma$-ray detector array must begin with consideration of the fluxes that are likely to exist. Two points of view prevail: the optimistic view that sources such as Cygnus X-3 exhibit "hard" photon spectra with integral spectral indices as small as 1 and the pessimistic view that the spectral indices are all significantly steeper with values of 2 or greater as typified by the Crab pulsar. The optimists support their case with the numerous claimed observations of Cygnus $\mathrm{X}-3$ over the energy range of $10^{11}-10^{16} \mathrm{eV}$ spanned by Cherenkov and extensive air shower detectors. Pessimists base their estimates on an interpolation between satellite data at lower energies and upper limits set by Cherenkov detectors or satellite data alone. As examples, the data for the Crab pulsar [1-11] and Geminga [12-18] are shown respectively in figs. 1 and 2 . Although the spectral index is close to one at lower energies the flux must fall much more rapidly above 1 GeV to be consistent with the detection limits set by the majority of the ground-based Cherenkov observations.

The case for a $1 / E$ energy dependence for sources such as Cyg X-3 and Her X-1 is weakened by a number of inconsistencies among the various results [19] despite the semblance of reasonable levels of statistical significance. Examples include conflicting claims for the $\gamma$-ray emission phase relative to the binary orbital period, pulsar periods inconsistent with X-ray data and signifi- cant muon fractions within extensive air shower events. None of these arguments can prove that Cyg X-3 is not the marvelous object that has been so widely advertised but it is surprising that a $\gamma$-ray flux which falls as $E^{-2}$ or faster [20] from $10 \mathrm{keV}$ to $1 \mathrm{GeV}$ abruptly flattens out at a threshold near $100 \mathrm{GeV}$, contrary to naive physical intuition.

In spite of these uncertainties, one must gamble on which kind of energy spectrum prevails since the choice completely determines the optimal experimental strategy. In the extreme case that the spectrum falls as $1 / E$, detection becomes easiest at the extensive air shower (EAS) threshold energy of $10^{14} \mathrm{eV}$ where the hadronic background is relatively smaller and can be substantially further reduced by vetoing muon-rich events. However, if the integral spectrum falls as $1 / E^{2}$ or faster, the counting rate in EAS arrays will drop below a few events per year. Under these conditions the Cherenkov detectors will be most effective at the lowest possible thresholds, $100 \mathrm{GeV}$ or less. The latter assumption is the guiding principle for the remainder of this article.

On the basis of the data shown in figs. 1 and $2 \mathrm{I}$ have adopted a flux sensitivity of $10^{-7} \gamma /\left(\mathrm{m}^{2} \mathrm{~s}\right)$ at $100 \mathrm{GeV}$ as a design goal for the next generation of Cherenkov air shower detectors. Of course, there is no guarantee that this will be sufficient to detect the Crab pulsar and Geminga but at least one might have a fighting chance. Such a sensitivity would also ensure detection of any source with spectral index of one or greater which could be observed above the $10^{-10} \gamma /\left(\mathrm{m}^{2} \mathrm{~s}\right)$ limit of the Chicago-Michigan electron/muon counter array [21] at $10^{14} \mathrm{eV}$. Achieving such a sensitivity is not an easy task. We will also assume that a $10^{-7} \gamma /\left(\mathrm{m}^{2}\right.$ s) flux must be detectable to a statistical significance of $5 \sigma$ within an observing time of $20 \mathrm{~h}$.

The only free parameters of the detector design are: (a) the effective sensitive area of the detector, $A$ (in $\mathrm{m}^{2}$ ); (b) the angular resolution of the system, $\mathrm{d} \Omega$ (in 


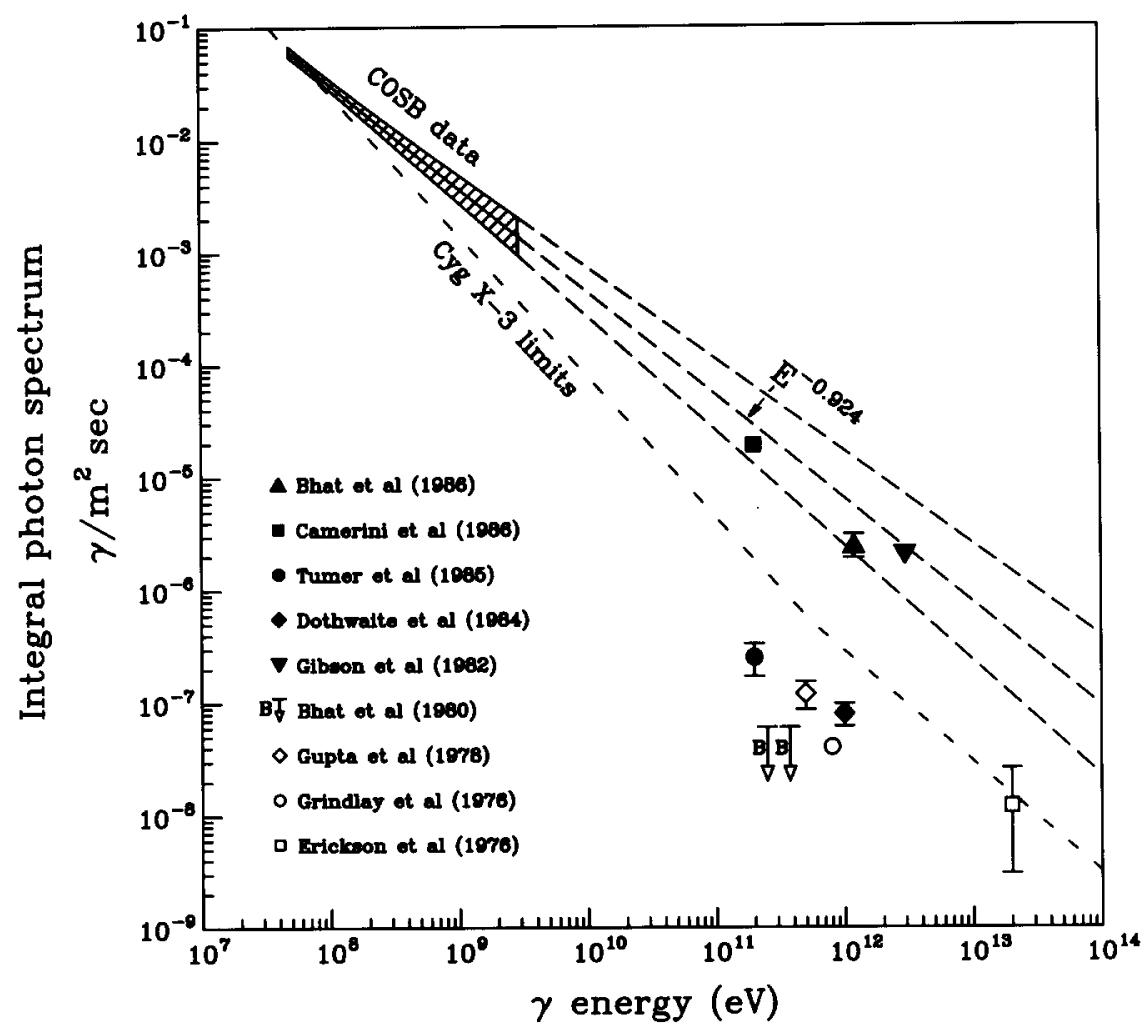

Fig. 1. The $\gamma$-ray flux from the Crab pulsar (PSR $0531+21$ ). The COSB data represents the integral of a power-law fit to the differential spectrum given by Clear et al. [1]. For comparison, the dotted line labeled "Cyg X-3 limits" approximately describes the measured fluxes or upper limits for this source.

$\mathrm{sr}$ ); and (c) the hadronic rejection factor, $R$.

In $20 \mathrm{~h}$ the number of $\gamma$-ray showers will be:

$N_{\gamma}=7.2 \times 10^{-3} \mathrm{~A}$,

while the background of hadron events $[22,23]$ will be:

$N_{\text {hadron }}=1.6 \times 10^{5} \frac{A \mathrm{~d} \Omega}{R}$.

(At these energies, a $200 \mathrm{GeV}$ hadron shower will produce about the same amount of light as a $100 \mathrm{GeV} \gamma$ shower.) For $5 \sigma$ significance the following inequality must hold:

$\frac{A R}{\mathrm{~d} \Omega} \geq 7.7 \times 10^{10} \mathrm{~m}^{2} \mathrm{sr}$

One may anticipate a detection area, $A=\pi(80 \mathrm{~m})^{2}$, and an angular resolution, $\mathrm{d} \Omega=\pi(4 \mathrm{mrad})^{2}$ so that a hadronic rejection factor greater than 190 must be realized. Finding a single method for vetoing hadronic showers to the level of 1 part in 200 is exceedingly difficult so the application of a number of independent selection criteria will be essential. The alternative of increasing the detection area has been suggested by Cawley [24] and Fry [25] in presentations at this workshop but the required number of independent light collectors appears formidable. A more serious objection is associated with the problem of signal detection with less than $1 \%$ signal-to-noise ratio. At this level, systematic effects are notoriously difficult to control. These arise from a large number of sources; for example, electronic gain variation with temperature, distant lightning, local variations in cloud conditions, and satellite glints. The problem is most severe if stellar sources only produce aperiodic bursts as claimed by several recent observations [19]. Since the angular resolution cannot be drastically improved beyond the $4 \mathrm{mrad}$ value assumed above, efficient hadronic shower rejection is the only path to an adequate detection system.

To understand the limits on the directional accuracy of the primary $\gamma$-ray requires some appreciation for the electromagnetic shower geometry. Fig. 3 is a schematic view of a shower developing obliquely in the atmosphere. For $100 \mathrm{GeV}$ primary energy, the longitudinal shower extent is short enough that the Cherenkov light wavefront can be considered approximately spherical in the forward direction within a few degrees of the primary direction. At the surface of the earth, the Cherenkov light will more or less uniformly illuminate a circular area with a radius of $125 \mathrm{~m}$. Beyond this region the Cherenkov flux will drop relatively slowly up to transverse distances of $1 \mathrm{~km}$ or more from the shower core. 


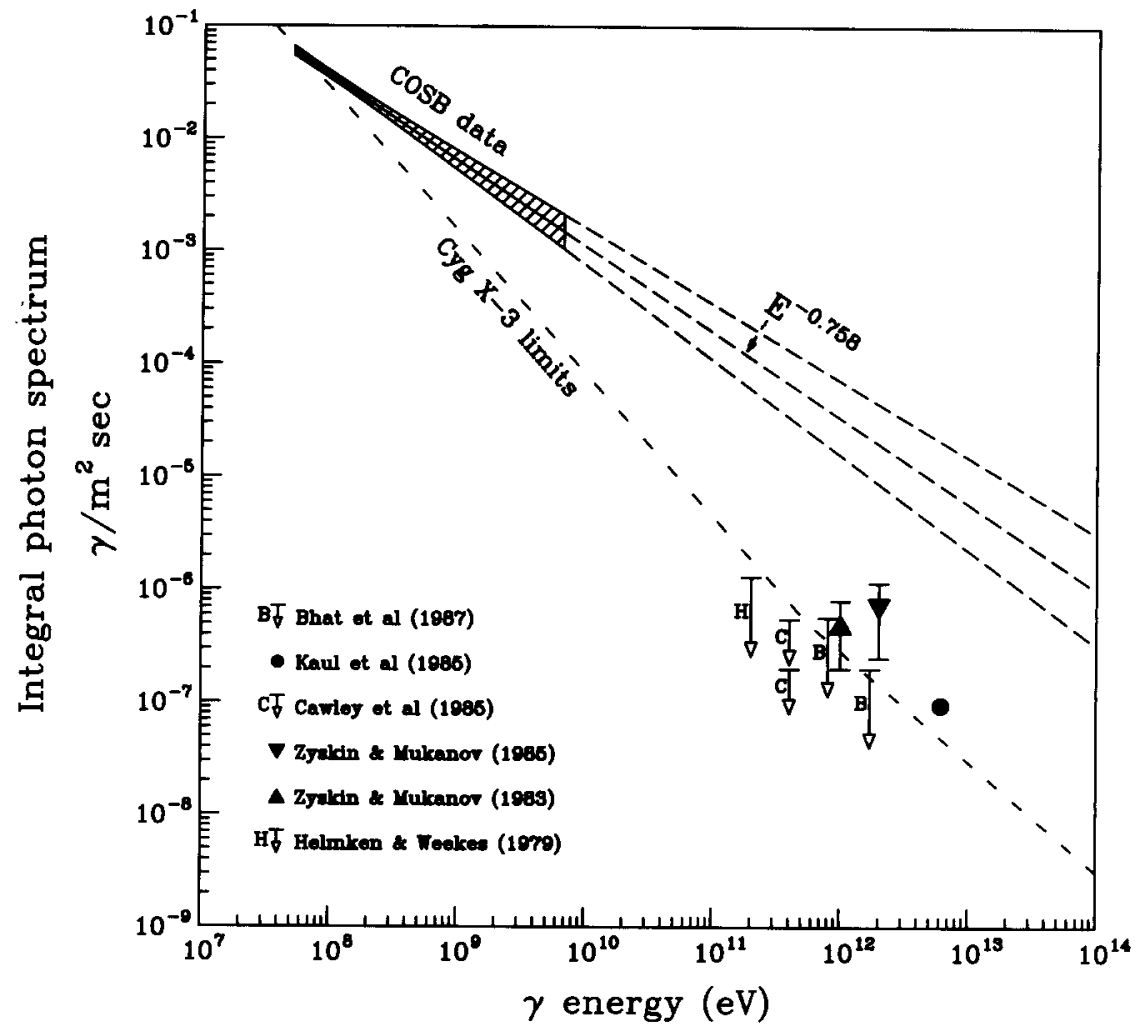

Fig. 2. The $\gamma$-ray flux from Geminga (2CG 195+04). The COSB data represents the integral of a power-law fit to the differential spectrum given by Caraveo et al. [12]. For comparison, the dotted line labeled "Cyg X-3 limits" approximately describes the measured fluxes or upper limits for this source.

Because the Cherenkov light pulse wavefront is not planar, estimates of the shower direction require two sets of measurements. One must determine the center of the light pool on the ground as well as the apparent position of the radiating electron/positron tracks overhead. The first measurement can be improved by sampling the light pool intensity with a number of detectors arranged on a uniform grid; the sky position can be obtained either by optical imaging of the Cherenkov light or by wavefront timing. Particularly if timing is used, the limiting accuracy for the primary $\gamma$-ray direction will be set by the error in determining the light pool center.

The characteristic Cherenkov light distribution within a $125 \mathrm{~m}$ radius is related to the dependence of the Cherenkov light cone for a relativistic particle as a function of atmospheric depth, shown in fig. 4. The tangent of this angle multiplied by the height above the surface yields the radius of the Cherenkov ring, drawn in fig. 5 for a surface elevation of $1700 \mathrm{~m}$, the altitude of our detector in Albuquerque. The radius rises to a maximum of less than $130 \mathrm{~m}$ at a depth of 3 radiation lengths and then linearly decreases. Since the shower maximum is near 5 radiation lengths, the Cherenkov light from all high momentum particles is constrained within the $125 \mathrm{~m}$ radius limit described above. Unfortunately, electromagnetic showers also produce large numbers of low energy electrons which Coulomb scatter to angles of $5^{\circ}$ or more. Monte Carlo simulations predict light distributions similar to the histogram in fig. 6 with half the light falling beyond $400 \mathrm{~m}$ from the shower core. These results are similar to curves obtained by Hillas and Patterson [26].

Since the rapid Cherenkov flux decrease at $120 \mathrm{~m}$ is characteristic of most events, a numerical experiment was performed to see how well the shower center could be determined. An array of 16 large concentrators was assumed to be located along the arms of a three-pointed star (like the Mercedes-Benz trademark) as shown in fig. 7. This geometric design was chosen to permit three independent measurements of the edge of the Cherenkov light density distribution. Light distributions were generated for a number of $100 \mathrm{GeV}$ electromagnetic showers arriving along the zenith direction aimed at the center of the array; for each event the light pool center was computed by finding the $120 \mathrm{~m}$ edge along each of the three arms. This somewhat idealized model indicated that a $2 \mathrm{mrad}$ angular resolution should be achievable at the cost of a large number of detectors and a relatively small active area. A more parsimonous 


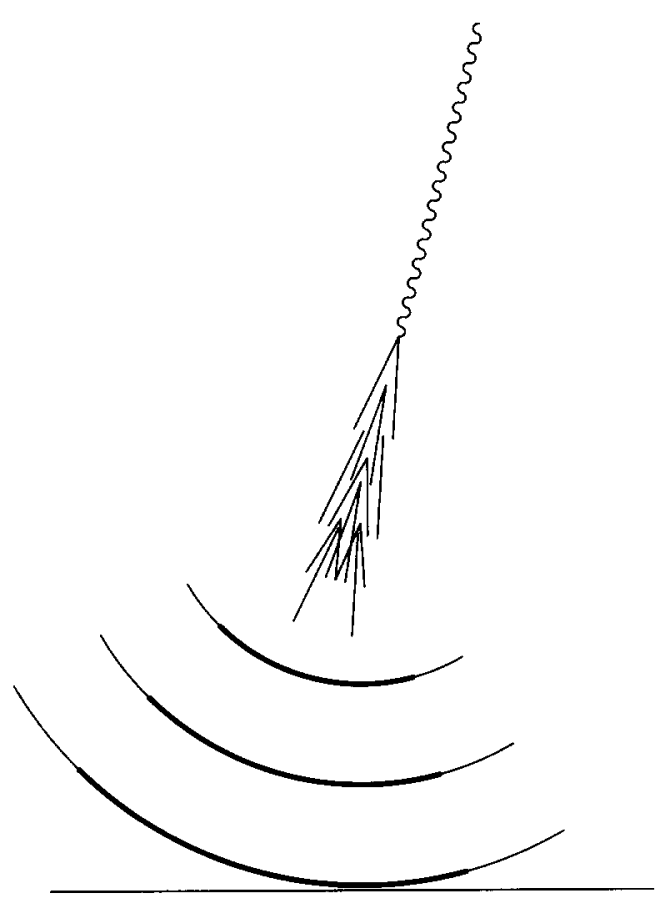

Fig. 3. Schematic view of an electromagnetic shower initiated by a high energy gamma ray. The Cherenkov light is emitted as a thin spherical shell of light in the forward direction. Reconstruction of the initial photon direction requires both the measurement of the Cherenkov light distribution on the surface and the apparent source location in the sky.

design with seven concentrators in a hexagonal array as proposed by Weekes [27] can probably interpolate the center to about half this accuracy and thus provide a

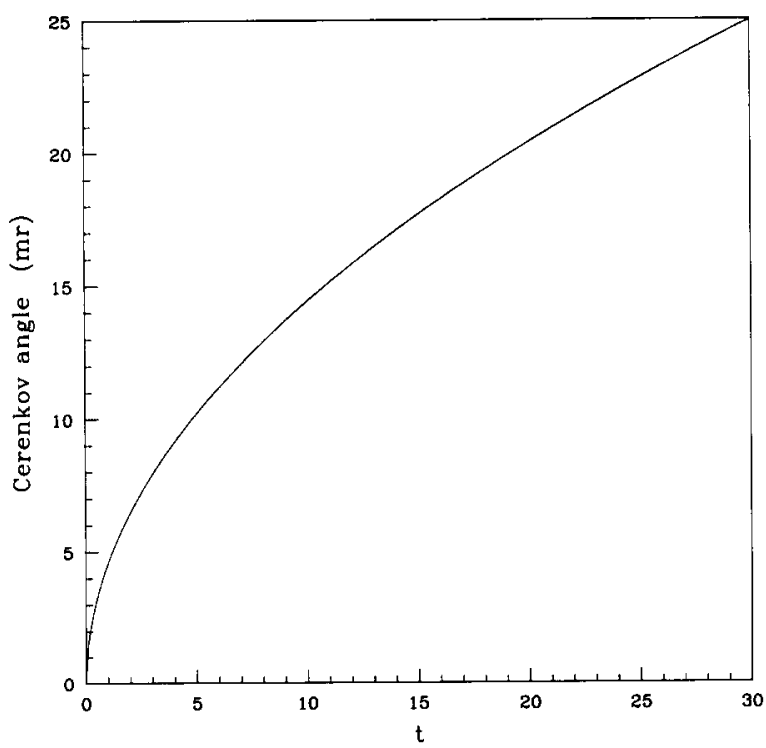

Fig. 4. The Cherenkov emission angle for a relativistic particle is plotted as a function of atmospheric depth, $t$, measured in radiation lengths. An isothermal atmosphere was assumed.

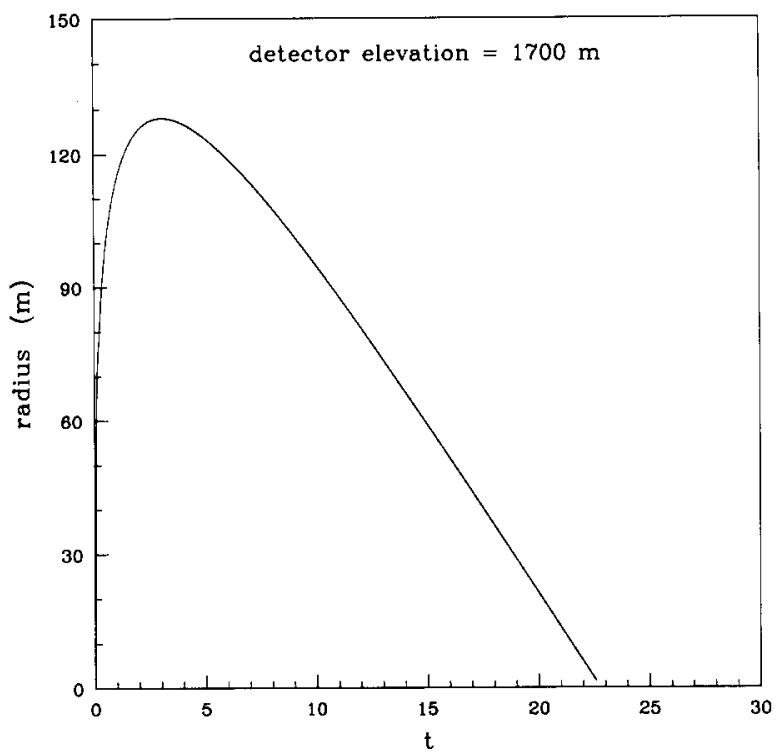

Fig. 5. The radius of the Cherenkov light cone at $1700 \mathrm{~m}$ altitude is plotted as a function of the atmospheric depth of the radiating particle measured in radiation lengths. An isothermal atmosphere was assumed.

shower direction with 4 mrad error. This basic array design of seven units will be assumed throughout the remainder of this paper.

Since angular resolution is intrinsically limited by the fluctuations of shower development, the only real hope for greater sensitivity lies in finding characteristic differences between photon and hadron initiated showers. Two methods have been described by e Weekes

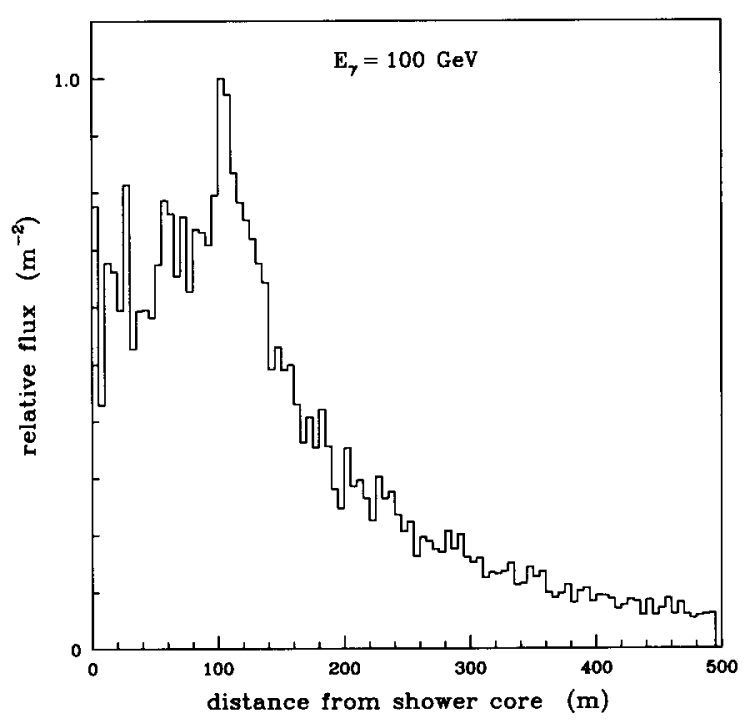

Fig. 6. The Cherenkov light flux density is plotted as a function of radial distance from the shower core for a typical Monte Carlo generated $\gamma$-ray event with $E_{\gamma}=100 \mathrm{GeV}$. 


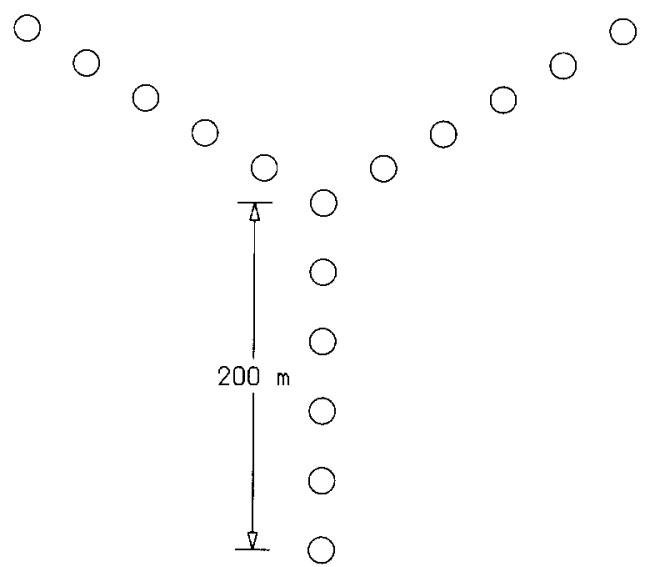

Fig. 7. A possible array of Cherenkov light detectors for obtaining $2 \mathrm{mrad}$ resolution for the initial photon direction. Each of the 16 circles is assumed to be a light collector with a diameter of $10-15 \mathrm{~m}$.

in his review talk [28] at this conference: fine grain imaging which his group is actively pursuing and far ultraviolet Cherenkov detection to veto penetrating showers, suggested by the Crimean group [29].

Hillas and Patterson have suggested the possibility that the time structure of Cherenkov light pulses might be somewhat different for photons and hadrons [26]. In earlier work at Mt. Hopkins, Jonathan Grindlay [30] had noted a stochastic behavior of light pulses from showers of predominately hadronic origin. The morphology of the pulse shapes consisted of an initial pulse followed by one or more after-pulses $20 \mathrm{~ns}$ or so later. If this pulse structure is related to particular features of hadronic shower development we must try to understand the physical causes to see if such effects might provide a useful discriminator of shower origin. From Monte Carlo simulation we know that the radiating electrons and positrons are distributed fairly close to the shower core. The radial distribution of the Cherenkov light source density is shown in fig. 8; a large fraction of the light is emitted within $40 \mathrm{~m}$ of the primary $\gamma$-ray trajectory. The longitudinal shower development can be approximated by the Rossi-Greisen calculations [31], as shown in fig. 9, which include the effective energy thresholds for Cherenkov radiation as a function of atmospheric depth. By convolving the transverse spatial structure, assumed to be Gaussian, and the longitudinal shower development, we computed the expected light arrival time distribution for various distances from the shower core, as shown in fig. 10. This procedure inevitably exaggerates the pulse width because correlations of multiple scattering and longitudinal depth are ignored; the true pulse widths are almost certainly shorter. We conclude that electromagnetic showers are not likely to generate wide Cherenkov light pulses with random secondary structure.
Similar arguments can also be made for hadronic showers but with less certainty. Hillas and Patterson studied this problem and concluded that the arrival time spread would be rather narrow. Two possibilities could contrive to generate a wider structure: either a substantial fraction of the shower energy is longitudinally propagated by subrelativistic protons $\left(E_{\mathrm{P}} \simeq 30\right.$ $\mathrm{GeV}$ ) or the transverse shower dimensions are large enough to impose substantially longer optical path lengths. The validity of Monte Carlo simulations of these effects is rather sensitive to the distribution of energy transfers which take place in high energy proton-nucleus interactions. Thus, the best test of this proposal is likely to be empirical.

Weekes has given a thorough description of the use of imaging to discriminate electromagnetic from hadron showers [28]. It also relies on the characteristically wider transverse dimensions of the hadronic distributions. Since this method is assured of providing some useful level of rejection, any future Cherenkov detection system will certainly contain imaging as an important feature.

Not much has been said about trying to veto low energy showers that contain particles reaching the earth's surface. The energy window in which this could work is not very broad because at low energies $(\simeq 100 \mathrm{GeV})$ very few muons are produced and at higher energies $(\simeq 1000 \mathrm{GeV})$ the electromagnetic showers will self-veto. Still, it is an interesting idea which might merit further consideration. This method depends on the atmospheric absorption of ultraviolet light by ozone in the wavelength band of $200-300 \mathrm{~nm}$ [32-34]. At the peak of the absorption spectrum $(250 \mathrm{~nm})$, the earth's atmospheric provides about 100 absorption lengths [35], mostly above

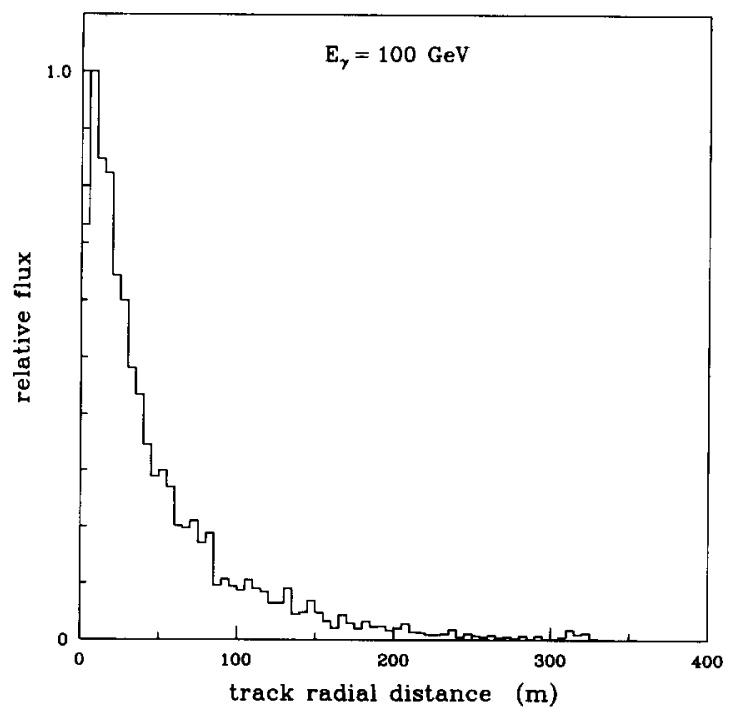

Fig. 8. The radial distribution of Cherenkov light emitting charged particle tracks for a $\gamma$-ray shower with $E_{\gamma}=100 \mathrm{GeV}$. 


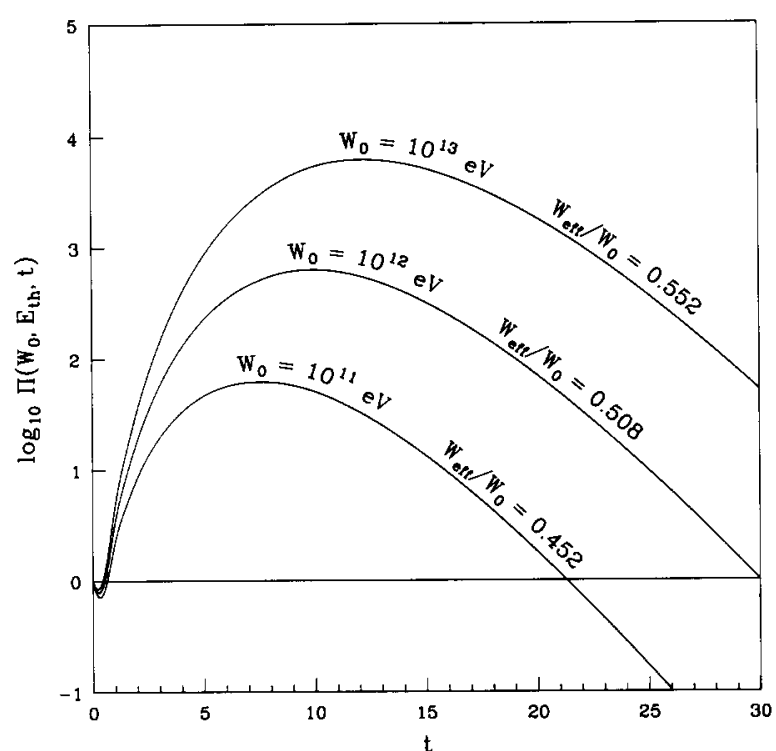

Fig. 9. The longitudinal distribution of Cherenkov light emitting charged particle tracks for showers initiated by $\gamma$-rays of energy $W_{0} . \Pi\left(W_{0}, E_{\mathrm{th}}, t\right)$ is the number of charged particles above the Cherenkov threshold at atmospheric depth, $t$. $W_{\text {eff }} / W_{0}$ is the ratio of the track length integral above Cherenkov threshold to the total track length.

$20 \mathrm{~km} \mathrm{[36]} \mathrm{but} \mathrm{near} \mathrm{the} \mathrm{surface} \mathrm{one} \mathrm{absorption} \mathrm{length}$ corresponds to a $1 \mathrm{~km}$ path. Thus one might selectively detect Cherenkov radiation from particles relatively low in the atmosphere, particularly muons. The technology for such measurements is not easy. Solar-blind photomultipliers with $\mathrm{Rb}-\mathrm{Te}$ photocathodes have long wavelength cutoffs near $300 \mathrm{~nm}$ but this may be insufficient to attenuate distant tracks and, furthermore, the quantum efficiency of these tubes is not high (5-10\%). Providing a large area coverage with an adequate quantum efficiency would appear to be a very costly endeavor. Such a technique might become feasible in the future to enhance the sensitivity of a detector based on either or both of the two stratagems outlined earlier.

At the moment, the only group which has really come to grips with the experimental necessities of $\gamma$-ray astronomy is the ASGAT collaboration led by Philippe Goret at SACLAY [37]. They plan to instrument seven solar concentrators on a $60 \mathrm{~m}$ hexagonal grid, very similar to Weekes' design of 1984. They hope to obtain a sensitivity of $5 \times 10^{-7} \gamma /\left(\mathrm{m}^{2} \mathrm{~s}\right)$ with a statistical significance of $5 \sigma$ within $10 \mathrm{~h}$ at a threshold energy of $100 \mathrm{GeV}$. Preliminary tests were conducted last year and funding for the full array has now been approved.

The cost of a new dedicated Cherenkov array depends strongly on the methods used to suppress the hadronic background. If imaging alone is used, the required pixel resolution and optical quality of the

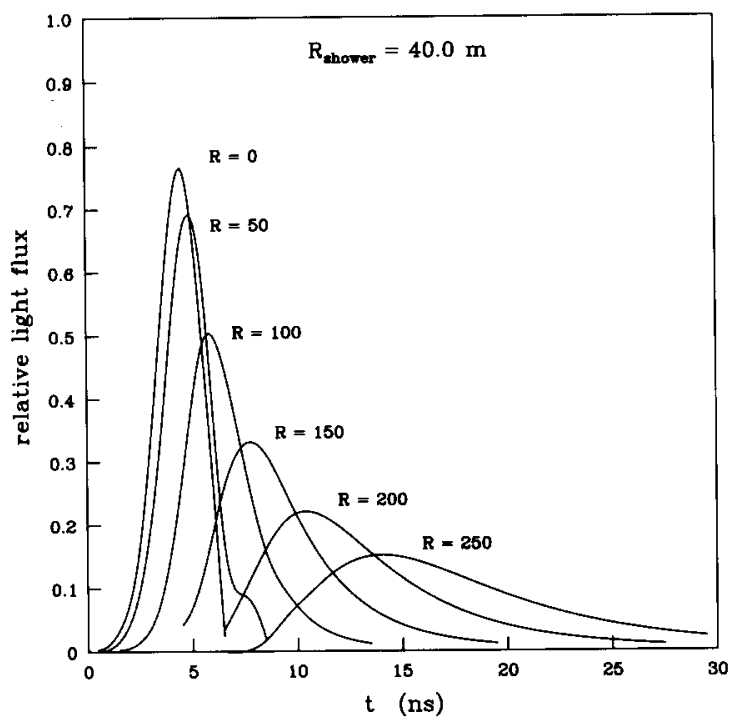

Fig. 10. The arrival time distributions expected for Cherenkov light from a $100 \mathrm{GeV}$ electromagnetic shower at several different distances from the shower axis. The transverse distribution of the shower was assumed to be Gaussian with $\sigma_{R}=40 \mathrm{~m}$.

concentrator may easily demand the 10 million dollar figure suggested by Weekes [28]. If pulse timing works at a level that reduces the demands on the imaging system one might be able to use commercially developed solar concentrators and fewer pixel elements for a total cost closer to 3 million dollars. Hopefully these issues will be better understood within the next year or so by work at Mt. Hopkins and in Albuquerque.

In summary I believe the entire field of ground-based $\gamma$-ray astronomy is a somewhat risky business. At the EAS threshold of $10^{14} \mathrm{eV}$ the proposed Chicago-Michigan array is likely to reach the claimed flux sensitivity of $10^{-10} \gamma /\left(\mathrm{m}^{2} \mathrm{~s}\right)$ but there is little guarantee of a source that can produce this much flux at such an energy. Certainly Cygnus X-3 is a dubious candidate at best.

At the Cherenkov detector threshold of about $10^{11}$ $\mathrm{eV}$, there are several sources which might be detectable if means can be provided to reduce the hadronic background by a factor of 200 or so. No such methods have been demonstrated to date. The alternative of building an array of a large number of small detectors for operation at intermediate energies of $10^{12}-10^{13} \mathrm{eV}$ faces severe difficulties in limiting time-varying systematic errors to less than $1 \%$. Very high energy $\gamma$-ray astronomy can potentially provide a unique and exciting window to our universe and to the behavior of matter under extreme conditions. It would be a shame to see this opportunity missed by failure to commit our various resources in a rational fashion to meet this challenge. 


\section{Acknowledgements}

The author gratefully acknowledges the advice and suggestions of G. Cassiday, C. MacCallum, D. Meyer, T. Tümer, T. Weekes, W. Wheaton, and J. van der Velde with especial thanks to J. Matthews for modifications of his shower Monte Carlo program that permitted calculations of the Cherenkov light flux. This work was supported by the U.S. Department of Energy contract DE-AC02-76ER01112.

\section{References}

[1] J. Clear, K. Bennett, R. Buccheri, I.A. Grenier, W. Hermsen, H.A. Mayer-Hasselwander and B. Sacco, Astron. Astrophys. 174 (1987) 85.

[2] P.N. Bhat, P.V. Ramanamurthy, B.V. Sreekantan and P.R. Vishwanath, Nature 319 (1986) 127.

[3] L. Resvanis, J. Learnel, V. Stenger, D. Weeks, J. Gaidos, F. Loeffler, J. Olson, T. Palfrey, G. Sembroski, C. Wilson, U. Camerini, J. Finley, W. Fry, M. Jaworski, J. Jennings, A. Kenter, R. Koepsel, M. Lomperski, R. Loveless, R. March, J. Matthews, R. Morse, D. Reeder, P. Sandler, P. Slane and A. Szentgyorgy, Very High Energy Gamma Astronomy, ed., K.E. Turner (Reidel, Dordrecht, 1987) pp. $155-158$.

[4] M.F. Cawley, D.J. Fegan, K. Gibbs, P.W. Gorham, R.C. Lamb, D.F. Liebing, P.K. Mackeown, N.A. Porter, V.J. Stenger and T.C. Weekes, 19th Int. Cosmic Ray Conf., La Jolla, CA, conference papers, vol. 1 (1985) 131.

[5] O.T. Tümer, W.A. Wheaton, C.P. Godfrey and R.C. Lamb, 19th Int. Cosmic Ray Conf. La Jolla, CA, conference papers, vol. 1 (1985) 139.

[6] J.C. Dothwaite, A.B. Harrison, I.W. Kirkman, H.J. Macrae, T.J.L. McComb, K.J. Orford, K.E. Turver and M. Walmsley, Astrophys. J. 286 (1984) L35.

[7] A.I. Gibson, A.B. Harrison, I.W. Kirkman, A.P. Lotts, J.H. Macrae, K.J. Orford, K.E. Turver and M. Walmsley, Nature 296 (1982) 833.

[8] P.N. Bhat, S.K. Gupta, P.V. Ramana Murthy, B.V. Sreekantan, S.C. Tonwar and P.R. Viswanath, Astron. Astrophys. 81 (1980) L3.

[9] S.K. Gupta, P.V. Ramana Murthy, B.V. Sreekantan and S.C. Tonwar, Astrophys. J. 221 (1978) 268.

[10] R.A. Erickson, R.K. Fickle and R.C. Lamb, Astrophys. J. 210 (1976) 539.

[11] J.E. Grindlay, H.F. Helmken and T.C. Weekes, Astrophys. J. 209 (1976) 592.

[12] P.A. Caraveo, G.F. Bignami, L. Vigroux, J.A. Paul and R.C. Lamb, Adv. Space Res. 3 (1984) 77.

[13] P.N. Bhat, N.V. Gopalkrishnan, P.V. Ramana Murthy, S. Swaminathan and P.R. Vishwanath, Astron. Astrophys. 171 (1987) 84.

[14] R.K. Kaul, H.S. Rawat, V.K. Sanecha, R.C. Rannot, M.L. Sapru, A.K. Tickoo, R.A. Qazi, C.L. Bhat, H. Razdan and
S.C. Tonwar, 19th Int. Cosmic Ray Conf. La Jolla, CA, conference papers, vol. 1 (1985) 165.

[15] M.F. Cawley, D.J. Fegan, K. Gibbs, P.W. Gorham, R.C. Lamb, D.F. Liebing, P.K. Mackeown, N.A. Porter, V.J. Stenger and T.C. Weekes, 19th Int. Cosmic Ray Conf. La Jolla, CA., conference papers, vol. 1 (1985) 173.

[16] Yu. L. Zyskin and D.B. Mukanov, 19th Int. Cosmic Ray Conf., La Jolla, CA, conference papers, vol. 1 (1985) 177.

[17] Yu.L. Zyskin and D.B. Mukanov, 18th Int. Cosmic Ray Conf., Bangalore, India, conference papers, vol. 1 (1983) 122.

[18] H.F. Helmken and T.C. Weekes, Astrophys. J. 228 (1975) 531.

[19] For a recent review of $\mathrm{TeV}$ and $\mathrm{PeV} \gamma$-ray stellar sources see the rapporteur talk by P.V. Ramana Murthy, ref. [3], pp. $39-51$.

[20] W. Hermsen, K. Bennett, J.B.G.M. Bloemen, R. Buccheri, F.A. Jansen, A. Mastichiadis, H.A. Mayer-Hasselwander, M.E. Özel, A.M.T. Pollock and A.W. Strong, Astron. Astrophys. 175 (1987) 141.

[21] James W. Cronin, presented at this Workshop (Workshop on Non-Accelerator Particle Physics, Rochester, NY, 1987).

[22] Richard E. Lingenfelter, Astrophys. Space Sci. 24 (1973) 83.

[23] A.M. Hillas, Phil. Trans. Roy. Soc. London A277 (1974) 413.

[24] Michael F. Cawley, these Proceedings (Workshop on Non-Accelerator Particle Physics, Rochester, NY, 1987) Nucl. Instr. and Meth. A264 (1988) 64.

[25] William F. Fry, presented at this Workshop.

[26] A.M. Hillas and J.R. Patterson, ref. [3], pp. 243-248.

[27] Trevor C. Weekes, Proc. of the Workshop on Cosmic Ray and High Energy Gamma Ray Experiments for the Space Station Era, Louisiana State University, Baton Rouge, LA (1985) p. 497.

[28] Trevor C. Weekes, ref. [24], p. 55.

[29] Yu.L. Zyskin, B.M. Vladimirsky, Yu.I. Neshpor, A.A. Stepanian, V.P. Fomin and V.G. Shitor, 20th Int. Cosmic Ray Conf., Moscow, USSR, conference papers, vol. 2 (1987) p. 342.

[30] J.E. Grindlay, Nuovo Cimento 2B (1971) 119.

[31] Bruno Rossi and Kenneth Greisen, Rev. Mod. Phys. 13 (1941) 240.

[32] Edward C.Y. Inn and Yoshio Tanaka, J. Opt. Soc. Am. 43 (1953) 870.

[33] M. Griggs, J. Chem. Phys. 49 (1986) 857.

[34] M. Ackerman, Mesospheric Models and Related Experiments, ed., G. Fioccio (Reidel, Dordrecht, The Netherlands, 1971) p. 149.

[35] Earl J. McCartney, Absorption and Emission by Atmospheric Gases: The Physical Processes (Wiley, New York, 1983) chap. 7.

[36] Louis Elterman and Robert B. Toolin, Handbook of Geophysics and Space Environments, ed., Shea L. Valley (McGraw-Hill, New York, 1965) chap. 7.

[37] Philippe Goret, private communication. 